\title{
Grußwort von Sigmar Gabriel
}

Die Welt hat sich verändert und der Wirtschaftsdienst mit ihr. 100 Jahre Wirtschaftsdienst sind deshalb auch ein Spiegel der wirtschaftspolitischen Debatten in unserem Land.

Schon bald nach seiner Gründung bot der Wirtschaftsdienst in den 1920er Jahren John Maynard Keynes, der mit seinen Thesen die klassische Nationalökonomie herausforderte, eine Plattform. Nach dem Krieg begleitete der Wirtschaftsdienst den Wiederaufbau und öffnete sich als eine der ersten wissenschaftlichen Zeitschriften der Debatte um die neue sozial-liberale Wirtschaftspolitik, in der auch Helmut Schmidt und Karl Schiller als Autoren zu Wort kamen. Als in den 1980er und 1990er Jahren Deregulierung und Privatisierung zum wirtschaftspolitischen Mainstream aufstiegen, blieb der Wirtschaftsdienst, nicht nur in der Rubrik „Zeitgespräch“, ein Ort der kontroversen Debatte. Im neuen Jahrtausend sind es insbesondere die Lehren aus der Wirtschafts-, Finanz und Staatsschuldenkrise, die die Diskussionen im Wirtschaftsdienst prägen. Auch greift er die hochaktuelle Diskussion über Ungleichheit und Wirtschaftswachstum auf. Kurzum: Wann immer Politik und Gesellschaft in den letzten 100 Jahren um den richtigen Weg in der deutschen Wirtschaftspolitik gerungen haben, der Wirtschaftsdienst war nicht nur als Chronist dabei, sondern bot widerstreitenden Meinungen auch ein Forum für die Debatte.

Diese Debatte brauchen wir heute so dringend wie damals. Auch wenn unser Land aktuell im Sommer 2016 stark dasteht, mit stabilen Wachstumsraten, Rekordbeschäftigung, steigenden Einkommen und sinkender Arbeitslosigkeit, müssen wir sehen, dass die mittelfristigen Herausforderungen für unser Land gewaltig sind.

Schon vor dem britischen Referendum war klar, dass Europa starken Fliehkräften ausgesetzt ist und unter den wirtschaftlichen und sozialen Ungleichgewichten zwischen florierenden und stagnierenden Mitgliedstaaten leidet. Hohe Arbeitslosigkeit und rückläufige Nachfrage, bedingt durch stagnierende Einkommen und rigorose Sparpolitik, gefährden den Zusammenhalt der Gesellschaften in einer Reihe von Mitgliedstaaten und schwächen die Wachstumspotenziale der Wirtschaft. Deshalb müssen wir fragen, wie der Stabilitäts- und Wachstumspakt seinem Namen wieder gerecht werden und für Wachstum und Wohlstand in allen Mitgliedstaaten sorgen kann. Wir brauchen dazu zusätzliche Investitionsimpulse auf europäischer Ebene. Eine gerechte Steuerpolitik, die im internationalen Kontext Steuervermeidung und Steuerbetrug effektiv bekämpft, könnte dafür die Spielräume schaffen.

Der demografische Wandel wird Deutschland härter treffen als viele andere Mitgliedstaaten. Deshalb ist die Frage der Sicherung unserer Fachkräftebasis durch die Mobilisierung inländischer Begabungsreserven und durch Zuwanderung für unsere Wirtschaft von zentraler Bedeutung. Gute Bildungspolitik und eine erfolgreiche Integration der Flüchtlinge in den Arbeitsmarkt werden darüber entscheiden, ob wir diese Herausforderung meistern.

Die Digitalisierung stellt unser industriell geprägtes Wirtschaftsmodell auf den Prüfstand. Wenn auch in Zukunft die besten Fahrzeugbauer und Fabrikausrüster der Welt in Deutschland und nicht in Kalifornien sitzen sollen, müssen wir dem Übergang zu Industrie 4.0 meistern. Nur mit einer modernen Industriepolitik, die gerade dem Mittelstand Orientierung in der digitalen Transformation gibt, werden wir uns im internationaIen Wettbewerb behaupten.

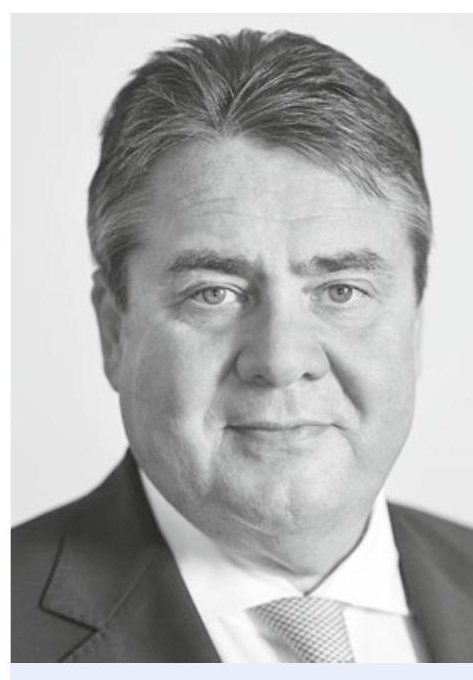

Sigmar Gabriel ist

Bundesminister für

Wirtschaft und Energie. 
Bei all diesen wirtschafts- und auch gesellschaftspolitischen Herausforderungen braucht die Wirtschaftspolitik wissenschaftlichen Rat über Handlungsoptionen und Entscheidungsalternativen. Die zunehmenden Möglichkeiten der Datenverarbeitung und neue Methoden, wirtschaftliche Entwicklungen mit Hilfe von mehr und besseren Daten gezielter nach Ursache-Wirkungs-Beziehungen zu analysieren, verbessern die Möglichkeiten für eine empirisch fundierte Wirtschaftsforschung. Konkrete Handlungsempfehlungen sind aber immer auch von unterschiedlichen Einschätzungen und Beurteilungen geprägt. Auch eine heute oft geforderte evidenzbasierte Wirtschaftspolitik bleibt auf politische Wertung und Entscheidung angewiesen.

Ich begrüße sehr, dass sich der Wirtschaftsdienst weiter mit der Lösung der drängenden Zukunftsfragen befasst - nicht nur in seiner Jubiläumsausgabe. Zu seinem 100-jährigen Jubiläum gratuliere ich dem Wirtschaftsdienst und seinem Redaktionsteam sehr herzlich und wünsche weiterhin viel Erfolg. Bleiben Sie sich treu!

Ihr
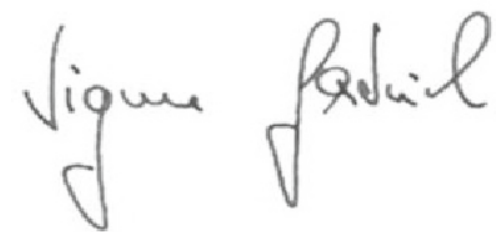